\title{
La politisation des étudiants antiautoritaires
}

Émergence des « espaces publics oppositionnels » et " mentalitéguérilla » à Berlin-Ouest

Marco Rampazzo Bazzan

\section{(2) OpenEdition}

\section{Journals}

Édition électronique

URL : http://journals.openedition.org/grm/269

DOI : $10.4000 / \mathrm{grm} .269$

ISSN : $1775-3902$

Éditeur

Groupe de Recherches Matérialistes

Référence électronique

Marco Rampazzo Bazzan, «La politisation des étudiants antiautoritaires », Cahiers du GRM [En ligne] 3 | 2012, mis en ligne le 29 mai 2012, consulté le 20 avril 2019. URL : http://journals.openedition.org/ grm/269; DOI : 10.4000/grm.269 


\title{
La politisation des étudiants antiautoritaires Émergence des « espaces publics oppositionnels » et « mentalité-guérilla » à Berlin-Ouest.
}

\author{
MARCO RAMPAZZO BAZZAN
}

Selon Bernd Rabehl, le mouvement étudiant des années soixante marque un changement qualitatif, voire même une rupture dans le rapport de la jeunesse intellectuelle avec les pouvoirs publics, puisque, pour la première fois dans l'histoire allemande, les étudiants contestent massivement les structures autoritaires de la société. Le jeune militant du Sozialistischer Deutscher Studentenbund (Union des étudiants socialistes) berlinois veut ainsi rappeler que, lors des conjonctures insurrectionnelles qui ont marqué le XXe siècle, la jeunesse intellectuelle allemande se mobilisant aux côtés des instances autoritaires et conservatrices avait joué un rôle contrerévolutionnaire ${ }^{1}$. Les corporations estudiantines avaient notamment constitué un bassin de recrutement pour la campagne impérialiste en 1914, pour la formation des Freikorps qui démantèleront les conseils des ouvriers et des soldats en 1919, et pour les corps spéciaux sous le IIIe Reich. Cependant, cette affirmation doit être relativisée et partiellement rectifiée, car, dans les années soixante, le mouvement n'est rallié que par une minorité, même si celle-ci est fortement engagée (comme ce fut le cas par ailleurs lors des émeutes de $1848)^{2}$. Cela dit, le mouvement étudiant allemand des années soixante est

${ }^{1}$ Bernd RABEHL, « Du mouvement antiautoritaire à l'opposition socialiste » in U. Bergmann, R. Dutschke, W. Lefèvre, B. Rabehl, La révolte des étudiants allemands, Paris, Gallimard, 1968 p. 325.

${ }^{2}$ Oskar Negt, Politik als Protest. Reden und Aufsätze zur anti-autoritären Bewegung, Frankfurt am Main, agi-buch-verlag, 1971, p. 50-51. 
surdéterminé par différents facteurs d'ordre historique, politique et social dont la complexité est souvent escamotée dans les études qui lui ont été consacrées $^{3}$. Par rapport aux autres mouvements étudiants à l'échelle européenne, les facteurs spécifiques de la mobilisation qui se développe en République Fédérale d'Allemagne sont: la continuité des structures autoritaires de la société de l'après-guerre avec celles du IIIe Reich, c'est-àdire la compromission d'une grande partie de la classe dirigeante, industrielle et politique, avec le nazisme ${ }^{4}$; la fin du 《miracle économique $»^{5}$ et la souveraineté réduite dont dispose le gouvernement à cause de l'ordre international issu de la deuxième guerre mondiale (la guerre froide). Cependant, cette contribution veut moins rendre compte d'une telle complexité que cerner l'effet, dans la formation de l'attitude et de la mobilisation « antiautoritaires» (essentiellement berlinoises), d'une série de microévénements qui structure cette séquence historique tout en restant surdéterminée par ces facteurs composant la conjoncture. Il s'agit d'analyser le processus de politisation du milieu étudiant berlinois sous conjoncture, c'est-àdire à partir des effets de ces micro-événements sur les acteurs au niveau de l'élaboration de leurs stratégies, et de l'impact des tactiques adoptées sur la ligne dynamique de la confrontation qu'ils engagent contre les autorités publiques et les forces conservatrices. Le but est de cerner une série de microévénements comme séquence d'effets de seuil provoquant des prises de conscience face à des impasses tactiques et découlant des rapports de force objectifs largement défavorables aux étudiants. Cette situation " minoritaire " les obligera à redéfinir constamment leurs stratégies afin de déjouer la répression dont ils font l'objet, en engendrant par là de nouvelles pratiques de lutte. L'insistance sur la nature de l'affrontement et sur ses mutations tout au long de la séquence nous permettra de montrer que, loin d'être l'issue inévitable de ce type de protestation, la dérive terroriste découle bien plutôt d'une crise politique que l'appareil répressif d'État et la presse conservatrice

${ }^{3}$ Lothar VOIGT, Aktivismus und moralischer Rigorismus: die politische Romantik der 68er Studentenbewegung Wiesbaden, Deutscher Universitäts-Verlag, 1991 ; Marica TolomelLI, "Repressiv getrennt" oder " organisch verbündet». Studenten und Arbeiter in der Bundesrepublik Deutschland und in Italien, Opladen, Leske \& Budrich, 2001.

${ }^{4}$ Christiane KoHSER-SPOHn, Mouvement étudiant et critique du fascisme en Allemagne dans les années soixante, Paris, L'Harmattan, 1999.

${ }^{5}$ Franz JÁNOSSY, Das Ende der Wirtschaftswunder. Erscheinung und Wesen der wirtschaftlichen Entwicklung, Frankfurt am Main, Neue Kritik, 1966. 
ont largement contribué à déterminer. Autrement dit, l'intransigeance de l'attitude des autorités publiques face à la contestation pousse les militants à forger une théorie adéquate à leur situation et à en changer les critères dans le processus de la lutte, jusqu'à théoriser une guérilla urbaine en République Fédérale d'Allemagne. Ce qui constituera pour certains d'entre eux la base idéologique qui les motivera à créer des cellules terroristes comme le Mouvement du 2 juin, les Tupamaros de Berlin-Ouest ou la Fraction de l'Armée Rouge (RAF).

L'enjeu théorico-politique de cette lutte est une mise en cause radicale des catégories de la pensée politique moderne qui constituent la base idéologique des sociétés occidentales. L'enseignement politique qu'en tirent les militants est que briser le consensus de la tolérance accordée par le pouvoir en place peut déclencher le déploiement de son appareil répressif selon des modalités autoritaires s'accompagnant d'une réduction progressive des droits civils. Cette mobilisation engage une lutte qui met en cause le paradigme politique moderne fondé sur la séparation entre théorie et pratique, c'est-à-dire, l'appareillage conceptuel et idéologique qui structure le couple individu-Etat, la réification des fictions de la représentation moderne héritées de la Révolution Française, tels le mandat libre et la verticalité corrélative de la relation politique et de sa reproduction - non seulement dans tout appareil intégré (parti, syndicat etc.), c'est-à-dire dans toute organisation admise et autorisée à agir politiquement sur la base de la séparation artificielle entre privé et public, mais aussi dans une grande partie des fractions oppositionnelles qui tendent à reproduire ces schémas dans leur organisation. D'un côté, afin de se conserver, les appareils intégrés répriment en leur propre sein toute dynamique non-organique, dissidente et créatrice de nouvelles subjectivités politiques (les lignes de fuite de Deleuze et Guattari). De l'autre, afin de subsister dans l'espace politique, toute spontanéité dissidente devient l'objet de la capture par la logique étatique qui impose aux militants l'alternative existentielle entre d'une part la réintégration sans appel ou la reproduction au sein des appareils constitués, et de l'autre leur neutralisation politique. Autrement dit, l'appareil d'État, répressif et idéologique, leur impose l'alternative entre la soumission aux « règles démocratiques » fixées par les autorités et la nécessité de s'engager à les affronter sur le terrain de l'illégalité en devenant par conséquent l'objet d'interpellations policières : tertium non datur. Et c'est exactement cette intolérance qui fait jouer un rôle fondamental à 
la réintroduction par Marcuse, dans son texte intitulé Tolérance répressive, de l'ancienne problématique d'un droit de résistance au sein des sociétés occidentales $^{6}$, ce qui conduira (contre les intentions de l'auteur et de la majorité des étudiants qui s'en inspire), par une série de transcodages événementiels, à l'ouverture d'un front de lutte armée au sein de la République Fédérale d'Allemagne.

La transformation de la structuration de l'espace public est à la fois un indice et un élément constitutif de la crise à l'origine de la protestation. Dans son article Über das Verhältnis zwischen Provokation und Öffentlichkeit, Oskar Negt, le futur théoricien de l'espace public oppositionnel, constate que la discussion, l'argumentation et la critique ne sont plus les facteurs qui forment l'opinion publique. Les moyens de communication ne servent désormais qu'à disposer les citoyens à acclamer l'un ou l'autre des représentants politiques ${ }^{7}$ et se transforment en de véritables outils de dépolitisation $^{8}$. Pour le dire avec les mots de Marcuse,

si le langage des politiciens tend à s'identifier à celui de la publicité (...) cette tendance semble indiquer dans quelle mesure la domination et l'administration cessent d'être des fonctions séparées et indépendantes dans la société technologique.

Negt souligne à ce propos que les moyens de communication servent à mobiliser des attitudes réactionnaires, a-politiques, qui trouvent leur ancrage dans le milieu petit-bourgeois, c'est-à-dire dans la sphère privée idéalisée comme domaine de Frieden, de la « paix» (église, famille) que l'Etat doit protéger et que les étudiants antiautoritaires menaceraient. Le but est d'immuniser certaines couches de la population face à toute proposition de changement et de les rendre imperméables à tout questionnement des valeurs fondant la société et des moyens mis en place pour leur réalisation concrète, c'est-à-dire, selon les militants, des pratiques autoritaires qui l'ordonnent et qui en permettent la reproduction en conservant ainsi sa hiérarchie ${ }^{10}$.

${ }^{6}$ «Je crois qu'il y a un "droit naturel" de résistance pour les minorités opprimées et étouffées, un droit d'utiliser des moyens illégaux si les moyens légaux se révèlent inadéquats » (Herbert MARCuSE, La tolérance répressive suivi de Quelques conséquences sociales de la technologie moderne, Paris, éditions Homnisphère 2008, p. 78).

${ }^{7}$ O. Negt, Politik als Protest, op. cit. p. 139.

${ }^{8}$ Ibid.

${ }^{9}$ Herbert Marcuse, L'homme unidimensionnel, Paris, Minuit, 1968 (1964), p. 128.

${ }^{10}$ O. NeGt, Politik als Protest, op. cit. p. 138. 
Or, le « refus absolu », que Marcuse emprunte à Blanchot ${ }^{11}$, deviendra la devise de la mobilisation antiautoritaire : les étudiants s'attaquent à la société tout entière devenue intolérable par son entreprise de blocage et de manipulation, et veulent s'organiser selon de nouvelles formes de lutte ${ }^{12}$. Ces dernières donnent vie à une mobilisation, une expérimentation collective qui veut échapper aux appareils de capture de l'État et aux formes de subjectivation que sa logique et son fonctionnement imposent ${ }^{13}$. De leur côté, les autorités publiques entament une escalade «hystérique» qui vise à marginaliser et à criminaliser les revendications des militants anti-autoritaires, afin de bloquer toute contamination subversive d'autres milieux et tout processus de solidarité pratique avec d'autres luttes à l'échelle nationale ou internationale. Cette marginalisation et cette criminalisation des minorités extra-parlementaires se structurent essentiellement selon le schéma amiennemi forgé par Carl $\mathrm{Schmitt}^{14}$, et réactivent certaines contradictions qui avaient fait surface de façon dramatique à l'époque de la république de Weimar et surtout des pratiques discriminatoires à l'égard des juifs que l'Allemagne avait connues sous le nazisme ${ }^{15}$. En reprenant l'analyse de Marcuse, on peut indiquer essentiellement deux piliers de l'ordre imposé comme étant sacré et " intouchable » au sein des démocraties occidentales de l'après-guerre : le progrès technique, et le communisme international, ce dernier remplaçant idéologiquement le fascisme comme ennemi intérieur ${ }^{16}$. Dans les pays du capitalisme avancé :

les anciens conflits au sein de la société se modifient sous la double (et mutuelle) influence $\mathrm{du}$ progrès technique et $\mathrm{du}$ communisme international. Les luttes de classe et l'examen des «contradictions impérialistes » sont différés devant la menace de l'extérieur. Mobilisée contre cette menace, la société capitaliste a une cohésion interne que les stades antérieurs de la civilisation n'ont pas connue. ${ }^{17}$

${ }^{11}$ H. Marcuse, L'homme unidimensionnel, op. cit. p. 279. Maurice Blanchot, « Le refus », dans Le 14 juillet, ${ }^{\circ} 2$, Paris, Octobre 1958.

${ }^{12}$ Cf. Séminaire du GRM, séance du 23 janvier 2010 : Section Allemagne/GRM Andrea_Cavazzini 23 Janvier 2010.pdf.

${ }^{13}$ Cf. Rudi DutschKe, Ecrits Politiques, Paris, Christian Bourgois, 1968, p. 66-68.

${ }^{14}$ O. Negt, Politik als Protest, op. cit. p. 106.

${ }^{15}$ Ibid. p. 13.

${ }^{16}$ Les deux étant amalgamés sous la catégorie ambiguë de "système totalitaire », ou encore de dictature.

${ }^{17}$ H. MARCuSE, L'homme unidimensionnel, op. cit. p. 47. 
Selon Marcuse, cette mobilisation permanente a également un effet économique positif et pacificateur : car « la mobilisation contre l'ennemi est un puissant stimulant de production et d'emploi, elle entretient un niveau de vie élevé $\rangle^{18}$. Or, les turbulences des années 1960 naissent des brèches exogènes qui avaient brisé ce cadre économique et idéologique (« le miracle économique » en RFA). La contestation s'attaque à la trahison des valeurs affichées par les démocraties occidentales que les étudiants voient comme étant en contradiction évidente avec les politiques de répression des mouvements de libération dans le tiers-monde (Vietnam), et avec la politique de complaisance vis-à-vis du bloc soviétique. Tout cela est perçu comme « intolérable » et pousse les étudiants à une mobilisation dont le développement se nourrit de l'affrontement avec l'appareil policier ${ }^{19}$.

\section{Le mur de Berlin. Période de préparation.}

Bernd Rabehl indique comme premier événement de la nouvelle phase de l'histoire de la jeunesse allemande la construction du Mur le 13 août $1961^{20}$. Rappelons-nous que Berlin n'était pas seulement l'ancienne capitale du Reich, mais qu'elle aurait dû devenir, selon le discours du gouvernement fédéral de l'après-guerre, la future capitale de l'Allemagne réunifiée. En dépit de cette déclaration, le mur est bientôt perçu par les militants de l'extrême gauche comme l'emblème de la coexistence conservatrice des deux blocs et comme l'indice de l'hypocrisie et de la vanité du blocage au sein de leurs frontières respectives face à toute dynamique émancipatrice et toute critique du statu quo. Son édification renforce les politiques conservatrices et favorise la criminalisation de toute protestation capable de mettre en cause le pouvoir en place. L'Allemagne se retrouve ainsi être le front d'un conflit international qui la dépasse. Réduit à un objet historique, ce pays voit se reproduire en son propre sein les contradictions du monde de l'après-guerre, dont Berlin et son mur deviennent ainsi l'emblème et le catalyseur. Dans son article publié dans le deuxième numéro d'Anschlag, revue de la Subversive Aktion, Rabehl conçoit le mur comme le symptôme du destin de la théorie marxiste et de sa

\footnotetext{
${ }^{18}$ Ibid.

${ }^{19}$ Cf. R. DutschKe, Ecrits Politiques, op.cit. p. 42 et p. 74-75.

${ }^{20}$ B. RABEHL, « Du mouvement antiautoritaire à l'opposition socialiste », op. cit. p. 329.
} 
dialectique en RDA et en Union Soviétique. Les États du « socialisme réel» constituent moins la négation du capitalisme que son corrélat essentiel. Les deux blocs se soutiennent mutuellement par une interaction (Wechselwirkung) qui renforce le pouvoir en place. Par conséquent, loin d'être une ligne de démarcation, le mur constitue alors le pilier d'un ordre politique et atteste l'interdépendance solidaire des deux géants économiques. Toutefois il catalyse également selon Rabehl les énergies révolutionnaires en vue de la réunification de l'Allemagne - une réunification qu'il ne peut par ailleurs imaginer que socialiste en raison du progrès économique affiché par la RDA ${ }^{21}$.

Or, le mur renforce l'intolérance vis-à-vis de toute dissension interne. Le seul espace de confrontation idéologique toléré est l'opposition frontale de façade des deux blocs mise en scène par la propagande. Sa construction provoque une exaspération de la dialectique du schéma ami/ennemi schmittien comme garantie de l'ordre interne des États. Le souci des autorités politiques tant de la RFA que de la RDA, est de réprimer toute critique contre le système des valeurs qu'elles sont appelées à représenter sur la scène politique internationale. A cette fin, leur mobilisation est totale et tout dissident est poussé à expérimenter et à mesurer ailleurs, plus précisément de l'autre côté du mur, son fantasme de liberté ${ }^{22}$. La réponse à toute critique interne est soit l'expulsion, soit la neutralisation politique. Les espaces sont bien striés et occupés tant militairement que idéologiquement. Cette situation a des répercussions sur l'Université Libre à Berlin-Ouest : les étudiants commencent à découvrir le caractère unilatéral de la liberté qu'elle prétendait pourtant incarner et défendre. Cela donne vie à une phase de mobilisation et d'autogestion où la lutte contre les autorités académiques se transforme rapidement en lutte contre l'ordre établi ${ }^{23}$.

Entre 1961 et 1964, c'est-à-dire dans la phase qui précède l'explosion du mouvement étudiant allemand, les militants subversifs (de la Subversive

${ }^{21}$ B. RABEHL, «Wie teuer ist die Mauer? (Vorläufige Thesen zum ökonomischen und ideologischen Ost-West-Geschäft)» in Frank Bölckelmann et Herbert Nagel (éd.), Subversive Aktion. Der Sinn der Organisation ist ihr Scheitern, Frankfurt am Main, Neue Kritik, 1976, p. 230-238.

${ }^{22}$ «Les journaux publient des lettres de lecteurs particulièrement agressives : la "population" exigerait de sévères mesures contre nous. "Il n'y a qu'à les renvoyer à l'Est, ces bandes de sauvages" » (R. DUTSCHKE, «Les étudiants antiautoritaires face aux contradictions présentes du capitalisme et face au tiers-monde », op. cit. p. 139).

${ }^{23} \mathrm{Cf}$. la reconstruction d'Uwe Bergmann in U. Bergmann, R. Dutschke, W. Lefèvre, B. Rabehl, La révolte des étudiants allemands, op. cit. p. 9-68. 
Aktion et d'autres groupuscules de gauche comme le club Argument) sont à la recherche d'un point de vue « tiers » afin de pouvoir questionner ce système de coexistence et d'interaction des deux blocs. De façon emblématique Rudi Dutschke, meneur du mouvement, écrit dans son journal intime que la lecture de Paul Sweezy lui a fait comprendre qu'il avait inconsciemment défendu un point de vue «chinois» dans son premier article pour Anschlag ${ }^{24}$. Cette remarque montre bien que, dans la quête de ce point de vue critique « tiers », les étudiants mobilisés font référence aussi bien à la position du parti communiste chinois contre Moscou et à la révolution culturelle, qu'aux luttes des pays du tiers-monde, notamment le Vietnam et Cuba. Mais, selon Dutschke, leur quête restait pourtant confuse et désespérée. L'époque Adenaeur, bien caractérisée par le président de la République Lübke et ses connivences avec le régime nazi-socialiste, ne laissait en effet aucun espoir à l'émancipation, et la promesse de la réunification était destinée à rester lettre morte, comme le montrait bien le refus obstiné des autorités fédérales de reconnaître la République Démocratique d'Allemagne. Dans Konkret, Ulrike Meinhof résumait ainsi, en 1965, les raisons politiques de ce refus :

$\mathrm{Du}$ point de vue de la politique intérieure, reconnaître la RDA impliquerait pour le gouvernement de Bonn de rompre le front d'unification anti-communiste et de perdre l'ennemi extérieur, qui nous unit tous. $^{25}$

Cela était impossible en raison du fait que des relations diplomatiques entre les deux Etats allemands auraient déstabilisé l'équilibre interne et international, et leurs principes de fonctionnement. Bref : pendant la première moitié des années soixante les cercles subversifs et les militants du SDS tâtonnent, impuissants, tout en cherchant des points d'appui extérieurs sur lesquels pouvoir territorialiser leur affect anti-autoritaire, afin d'occuper un espace d'existence et de briser l'univers unidimensionnel analysé par Marcuse, dans lequel ils se trouvaient piégés. Dans ce contexte, les analyses de la politique internationale par Dutschke et Rabehl, qui font l'objet de critiques même au sein de la micro-cellule berlinoise de la Subversive Aktion, paraissent être l'indice de cette impasse politique, tout en témoignant pourtant de leur

${ }^{24}$ Rudi DutschKe, Jeder hat sein Leben ganz zu leben, Die Tagebücher 1963-1979. Herausgegeben von Gretchen Dutschke, Köln, Kiepenheuer et Witsch Verlag, 2003, p. 20-21.

${ }^{25}$ Ulrike Marie MeInHOF, Die Würde des Menschen ist antastbar, Berlin, Wagenbach, 1980 p. 65 . 
ambition $^{26}$.

Dans le premier numéro d'Anschlag, Rudi Dutschke affirme :

Il n'y a pas encore de socialisme sur la terre. Le socialisme demeure une catégorie de ce qui-n'est-pas-encore (Noch-Nicht-Sein), que les forces révolutionnaires doivent nécessairement réaliser socialement à l'échelle mondiale. ${ }^{27}$

Il exprime par là la volonté de renouer avec la tradition révolutionnaire (sous l'influence trotskiste) qui avait échoué tant à l'Ouest, où la politique de la social-démocratie avait ouvert le chemin au nazi-socialisme, qu'à l'Est, où la Révolution d'octobre avait dégénéré dans le stalinisme. Dutschke soutient ainsi avec force que l'Union Soviétique ne peut pas se borner à de simples déclarations contre le stalinisme, mais qu'elle aurait dû changer radicalement les pratiques politiques qui s'étaient établies et consolidées sous l'ancien chef. En revanche, il pose comme question capitale en Occident la nécessité de faire renouer la théorie sociale critique avec une pratique politique conséquente. Dutschke dénonçait l'incapacité des intellectuels de gauche - qu'il juge par ailleurs brillants - à assumer les conséquences pratiques découlant de leurs critiques contre la société :

Nous nous demandons comment ces penseurs extraordinaires [Adorno, Horkheimer, Habermas, Bloch etc.] peuvent continuer à maintenir cette séparation incompréhensible entre être et penser, entre théorie et praxis dans la condition actuelle de la République Fédérale Allemande. ${ }^{28}$

Dutschke et ses camarades ont recours aux auteurs dissidents comme Trotsky, Boukharine, R. Luxembourg, qui avaient payé leur cohérence au prix de leur vie. Ils refusent les dogmes de la Troisième Internationale et préconisent un marxisme conçu comme

une science créatrice, qui doit se confronter avec toute réalité nouvelle sur la base de la méthode critique qu'est la dialectique et qui doit forger avec celle-ci les catégories pour une nouvelle compréhension de

${ }^{26}$ Bernd RABEHL, «Sozialimperialismus und Sozialdemokratie» in F. Bölckelmann et H. Nagel (éd.), Subversive Aktion op. cit. p. 174-178; Rudi DuTschKE, «Die Rolle der Antikapitalistischen, wenn auch nicht sozialistischen Sowietunion für die marxistischen Sozialisten in der Welt » in F. Bölckelmann et H. Nagel (éd.), Subversive Aktion op. cit. p. 169174.

${ }^{27}$ Ibid., p. 169.

${ }^{28}$ R. DutschKe, Diskussion: das Verhältnis von Theorie und Praxis in F. Bölckelmann et H. Nagel (éd.), Subversive Aktion, op. cit. p. 190-195. 


\section{l'actualité concrète ${ }^{29}$.}

On voit bien que le questionnement des jeunes militants porte sur le passage du socialisme utopique au socialisme scientifique théorisé par Marx lui-même. Ce type de problématique ouvre un front de lutte interne à la gauche et, en ce qui concerne cette séquence, un conflit générationnel entre intellectuels-professeurs et étudiants, dont les moments le plus significatifs seront l'accusation de volontarisme et de fascisme de gauche que Habermas adressera à Dutschke en $1967^{30}$ et le recours de la part d'Adorno aux forces de l'ordre pour « libérer » l'Institut de critique sociale de Francfort, qui était occupé par nombre de ses étudiants. Parmi eux : Hans-Jürgen Krahl, dont les écrits auront un impact bien au-delà des frontières allemandes, tout spécialement en Italie.

Or, le problème des étudiants est de trouver une scène, un espace public où faire entendre leur critique de la société unidimensionnelle; et le seul espace à leur disposition, et qu'ils réussiront effectivement à investir, c'est la rue. Comme le soulignait Ulrike Meinhof à l'époque de son succès médiatique comme rédactrice de Konkret :

je ne retiens guère les rues comme un lieu particulièrement approprié pour manifester ses idées. Mais s'il ne nous reste pas autre chose, c'està-dire, si on n'a pas l'occasion de parler à la télévision et d'expliquer au moins deux ou trois fois par semaine exactement ce qu'on a à dire, si l'on ne dispose pas des millions de Springer, si lorsqu'on veut organiser une manifestation publique on tombe toujours sur des interdictions, alors je suis absolument persuadée que le fait qu'il y ait des gens qui malgré toutes ces interdictions occupent et utilisent le seul espace public encore à disposition qu'est la rue pour rendre publiques leurs idées, est tout à fait démocratique. ${ }^{31}$

Les protestations des rues marquent cette nouvelle subjectivité dissidente et deviennent ainsi le lieu d'expérimentation de nouvelles formes de lutte et de politisation. C'est dans la bataille des rues que les militants subversifs territorialisent leur élan révolutionnaire et construisent l'espace de

${ }^{29}$ R. DutschKe, «Die Rolle der Antikapitalistischen, wenn auch nicht sozialistischen Sowietunion für die marxistischen Sozialisten in der Welt » in F. Bölckelmann et H. Nagel (éd.), Subversive Aktion op. cit. p. 171.

${ }^{30}$ Voir J. Miermeister (éd.), Geschichte ist machbar. Texte über das herrschende Falsches und die Radikalität des Friedens, 1980, Berlin, Wagenbach, 1991, p. 82.

${ }^{31}$ Voir Urlicke MeINHOF : http://www.vidivodo.com/211473/ulrike-meinhof-raf 
leur existence politique. Comme le rappelle Oskar Negt :

Là où la conscience historique se forme dans la bataille pratique, se dissout le système des catégories transmises sous forme réifiée (...); il devient donc clair que les périodes révolutionnaires et postrévolutionnaires développent leurs concepts propres de politique et de protestation. $^{32}$

C'est dans les rues de Berlin-Ouest que prendra forme cette nouvelle subjectivation politique subversive et solidaire entre les militants métropolitains et les révolutionnaires du tiers-monde dont nous sommes toujours les héritiers.

\section{La manifestation contre Tschombé : la fonction du tiers-monde.}

La manifestation berlinoise contre Tschombé, Président du Conseil congolais soupçonné d'être impliqué dans le meurtre de Patrice Lumumba, constitue un événement majeur dans le procès de politisation des étudiants berlinois. Dutschke et Rabehl considèrent cette manifestation comme l'acte fondateur et constituant du mouvement, parce qu'elle forge les traits qui vont définir le mouvement antiautoritaire. Rabehl la définit rétrospectivement comme la «fin de la période de préparation $»^{33}$. Dans la manifestation contre Tschombé, les mots d'ordre du deuxième numéro d'Anschlag, paru quelques semaines auparavant, trouvent leur réalisation. À la question : "Pourquoi des Anschläge ?» la rédaction avait répondu que l'intention n'était pas de « renforcer le sentiment jouissif du savoir ». En rompant ainsi avec la critique radicale professée par le personnel enseignant dans les Universités, qu'ils jugent « demeurer (verharrt) dans la passivité et refuser la véritable critique comme identité de la théorie et de la pratique », leur analyse veut constituer « un tremplin (Sprungbrett) pour attaquer la réalité ${ }^{34}$. Dutschke voit le tournant de cette manifestation dans la décision collective de la rendre illégale et de surmonter ainsi les règles du jeu de la démocratie occidentale sur le plan pratique. C'est par cette décision que les manifestants se découvrent liés par

\footnotetext{
${ }^{32}$ O. Negt, Politik als Protest, op. cit. p. 71.

${ }^{33}$ B. RABEHL, « Du mouvement antiautoritaire à l'opposition socialiste », op. cit. p. 331.

${ }^{34}$ F. Bölckelmann ; H. Nagel (éd.), Subversive Aktion, op. cit. p. 229.
} 
une appartenance commune sur le plan matériel. En sautant par-dessus les barrages de police, les acteurs de la gauche berlinoise surmontent leur méfiance réciproque. Cette manifestation constitue un véritable apprentissage collectif de nouvelles pratiques de lutte qui sera le moteur de la dynamique antiautoritaire $^{35}$. Le choix de l'illégalité s'impose comme forme de protestation. Selon Rabehl, ce jour-là l'opposition de gauche avait appris : qu'il ne suffisait visiblement plus d'organiser des manifestations qui ne trouvaient aucun écho dans la société ou qu'on pouvait taxer de manœuvres subversives d'agents soudoyés». Il fallait briser «les règles du jeu» afin de contraindre les autorités, les partis politiques et les syndicats, à prendre position sur les contenus des protestations ${ }^{36}$.

En convoquant Deleuze et Guattari nous pourrions ainsi dire que BerlinOuest devient la surface d'émergence du « natal ». Selon les auteurs de Mille Plateaux, le «natal» est en effet la composition de l'inné et de l'acquis, de laquelle vient l'affect qui le caractérise comme étant «toujours perdu, retrouvé », ou tendant « vers une patrie inconnue ${ }^{37}$. Dans le cas de Dutschke et Rabehl l'émergence du natal est l'espace de réalisation du véritable socialisme dont ils avaient expérimenté l'impossibilité dans les deux Républiques allemandes (tous les deux avaient grandi en RDA). Cette émergence se laisse caractériser comme un « devenir-allemand », c'est-à-dire ni de l'Ouest, ni de l'Est, ni seulement chrétien, ni seulement socialiste, mais " entre-deux ", projection vers une patrie inconnue et perdue, vers une Allemagne réunifiée véritablement socialiste. Dans la perspective des militants, les rues de Berlin-Ouest - la « vitrine du monde libre »-deviennent le laboratoire d'une nouvelle société, émancipée de la guerre froide et de ses logiques répressives, le sol sur lequel bâtir leur utopie. Cette projection " consiste en un décodage de l'innéité et une territorialisation de l'apprentissage, l'un sur l'autre, l'un avec l'autre $»^{38}$. On pourrait cerner l'innéité comme une certaine idéologie petite-bourgeoise et l'apprentissage comme l'expérimentation de nouvelles pratiques dans les rues. Le constat de Dutschke qu'il « n'y a pas de socialisme sur la terre » signifie alors, pratiquement, revendiquer un «entre-deux», un espace qui n'existe pas encore, que les

${ }^{35}$ R. DutschKe, «Les étudiants antiautoritaires face aux contradictions présentes du capitalisme et face au tiers-monde », op. cit. p. 136-138.

${ }^{36}$ B. RABEHL, « Du mouvement antiautoritaire à l'opposition socialiste », op. cit., p. 337.

${ }^{37}$ G. Deleuze et F. Guattari, Mille Plateaux, Paris, Minuit 1980, p. 410.

${ }^{38}$ Ibid. 
étudiants antiautoritaires projettent sur Berlin-Ouest, la ville que la guerre froide a séparée de sa partie orientale et coupée du monde, que Dutschke théorise comme future république des conseils, comme ville libre et clef de voûte d'un nouvel ordre social ${ }^{39}$.

Selon Oskar Negt,

l'idée d'auto-gouvernement par des conseils fait surface régulièrement lors de crises du système de domination parce que ce dernier n'arrive plus à satisfaire les intérêts de la majorité. ${ }^{40}$

Dans cette réactivation spécifique, elle relève de sentiments antibureaucratiques et antiautoritaires que partagent aussi des couches minoritaires du mouvement ouvrier. « Là où s'établissent des conseils à l'heure actuelle, ils se constituent toujours sous la forme de l'auto-gestion socialiste », ce qui signifie vouloir en finir avec la politique comme une sphère séparée de la division du travail dans la société. Et celui qui soutiendrait qu'une telle autogestion est irréalisable doit simplement renoncer à l'espoir de vivre dans une société véritablement socialiste et assumer qu'il a accepté que les rapports de dépendance produits par le système de domination actuel soient devenus « naturels $»^{41}$.

Mais la conception de Berlin comme république des conseils veut également dire autre chose : que le véritable socialisme, à la manière du Prince machiavélien interprété par Althusser, se donne « dans le vide » d'une décision à prendre ${ }^{42}$. La question devient alors celle de la réunion de toutes les forces disponibles pour lui faire voir le jour. C'est à partir de là que s'articule « la fonction du tiers-monde ». En effet, les mouvements de libération du tiersmonde deviennent pour les étudiants antiautoritaires le point d'appui d'une territorialisation de la ville. Cette territorialisation passe par un décodage subversif de l'Université libre qu'ils veulent transformer progressivement en une Université critique et oppositionnelle, et par une occupation nomade de la ville à travers des manifestations contre la guerre au Vietnam et contre les

${ }^{39} \mathrm{Cf}$. R. DutschKe, Ecrits politiques, op. cit., p. 70.

${ }^{40}$ O. Negt, Politik als Protest, op. cit., p. 136-137

${ }^{41}$ Ibid.

42 «Cette nouvelle négation [celle du cycle comme tel] est donc très particulière et c'est pourquoi je ne lui ai pas donné la forme d'une quatrième thèse, mais j'ai parlé d'une position de Machiavel. Dans cette position, il y a un écart significatif, un vide, un saut dans le vide théorique, une anticipation » (Louis AlthusSER, Écrits philosophiques et politiques, éd. établie et présentée par F. Matheron, Paris, Stock-I.M.E.C., vol. II, 1995, p. 91). 
interdictions des manifestations qui se succéderont tout au long de cette séquence. La « fonction tiers-monde » donne aux militants une base matérielle et le sentiment d'appartenir à un mouvement révolutionnaire international. Selon Dutschke :

Le matérialisme historique se doit de dégager la fonction du tiersmonde, en tant qu'élément constituant de la révolutionnarisation du monde tout entier. ${ }^{43}$

Rabehl explique qu'ils trouvent à ce moment précis une médiation (Vermittlung) entre leurs actions dans les métropoles et les guerres de libération. En suivant Lénine et Trotsky, ils interprètent le concept de totalité hégélien comme histoire universelle (Weltgeschichte) et

les étapes «pacifiques » entre les crises du système bourgeois devinrent les périodes de préparation pour l'avant-garde révolutionnaire.

Ils voulaient répondre à «l'offensive de l'impérialisme» au Congo, à Saint-Domingue, au Vietnam par « une contre-offensive dans les pays hautement industrialisés et capitalistes $»^{44}$. Selon Negt, la protestation métropolitaine solidaire des révoltes sociales dans le tiers-monde avait permis de dévoiler les rapports de domination réifiés dans les sociétés occidentales. Loin de se réduire à une simple " solidarité des sentiments », cette dynamique forge bien plutôt une morale politique dans laquelle l'élément de la protestation, c'est-à-dire l'affect anti-fonctionnel en tant que contenu inconditionné des revendications politiques, s'articule à l'évidence de l'oppression matérielle dans le contexte colonial, une oppression qui n'était plus visible dans les métropoles. C'est sur cette base affective que se fonde le «refus absolu " théorisé par Marcuse. Les étudiants antiautoritaires ne peuvent pas « accepter les plaintes contre l'ethnocide perpétré par le IIIe Reich sans les transformer en action contre tout ethnocide actuel $»^{45}$.

Mais les luttes dans le tiers-monde donnent également aux étudiants des nouveaux modèles pratiques et des nouvelles orientations théoriques. Ils opposent les intellectuels de gauche - qui les avaient taxés de fascistes de gauche - aux militants et intellectuels impliqués dans les guerres de guérilla. Ils trouvent chez Frantz Fanon, Che Guevara, Mao et Régis Debray un autre

${ }^{43}$ R. DutschKe, «Les étudiants antiautoritaires face aux contradictions présentes du capitalisme et face au tiers-monde », op. cit., p. 150.

${ }^{44}$ B. RABEHL, « Du mouvement antiautoritaire à l'opposition socialiste », op. cit., p. 336.

${ }^{45}$ O. NeGt, Politik als Protest, op. cit. p. 35 
modèle de conduite, l'union de la théorie et de la pratique qu'ils voulaient traduire et incarner dans les métropoles. Les étudiants forgent une autre idée du militantisme, théorisent «l'homme nouveau », s'engagent dans la lutte en s'appropriant les élaborations théoriques de ces nouveaux guides au point de se fantasmer, parfois avec des dérives tragiques, comme " guérilleros urbains ", « colonisés » des métropoles combattant dans un front international de lutte ${ }^{46}$.

Dans cette perspective, nous pouvons dire qu'ils essaient de répondre à l'appel que Fanon lançait à la fin du premier chapitre de Les Damnés de la terre : pour faire triompher l'homme partout, le tiers-monde «n'entend pas organiser une immense croisade contre l'Europe »; mais à cette fin « il faudrait d'abord que les masses européennes décident de se réveiller, secouent leurs cerveaux et cessent de jouer au jeu irresponsable de la Belle au bois dormant $»^{47}$. Or, ce texte, dont la première partie fut traduite en allemand dans le deuxième numéro de Kursbuch, devient une source d'inspiration pour les étudiants et une manière de théoriser leur lutte ${ }^{48}$. Les militants antiautoritaires articulent leur affectivité et leur expérience à celles du colonisé décrites par Fanon. L'affect anti-fonctionnel dont parle Negt peut s'articuler à l'attitude du colonisé : «Dans le monde colonial, l'affectivité du colonisé est maintenue à fleur de peau comme une plaie vive ${ }^{49}$. Pour Dutschke, le colonisé est « aujourd'hui un zoon politikon au sens le plus plein du terme $»^{50}$. La figure de l'universitaire européen de gauche se rapprochait sinistrement de celle de l'intellectuel colonisé que Fanon décrit comme « un vulgaire opportuniste » qui privilégie le détail et en arrive à oublier la défaite du colonialisme : 《l'objet même de la lutte ${ }^{51}$. Cette solidarité avec le colonisé produit également une "colonisation intérieure » perçue par les étudiants dans la manière où les traite la presse. La manifestation contre Tschombé suscite en effet l'attention médiatique recherchée pendant longtemps par les militants de

${ }^{46}$ B. RABEHL, « Du mouvement antiautoritaire à l'opposition socialiste », op. cit. p. 337 et R. DutschKe, «Les considérations historiques de la lutte internationale por l'émancipation » in U. Bergmann, R. Dutschke, W. Lefèvre, B. Rabehl, La révolte des étudiants allemands, op. cit. p. 199.

${ }^{47}$ Frantz FAnON, Les Damnés de la terre, Paris, La Découverte, 2002 (1961), p. 103.

${ }^{48}$ R. DutschKe, «Les étudiants antiautoritaires face aux contradictions présentes du capitalisme et face au tiers-monde », op. cit. p. 149.

${ }^{49}$ F. FANON, Les Damnés de la terre, op. cit. p. 51.

${ }^{50}$ R. DutschKe, «Les étudiants antiautoritaires face aux contradictions présentes du capitalisme et face au tiers-monde », op. cit., p. 149.

${ }^{51}$ F. FAnON, Les Damnés de la terre, op. cit., p. 51. 
l'extrême gauche : le lendemain, leur action fait la une de tous les journaux. Mais ce gain de visibilité médiatique ne fait qu'ouvrir un front de lutte contre l'opinion publique formée par le Groupe-Springer et les forces de l'ordre. Fanon disait que :

Dans la guerre d'Algérie par exemple les reporters français les plus libéraux n'ont pas cessé d'utiliser des épithètes ambiguës pour caractériser notre lutte. Quand on leur en fait le reproche, ils répondent en toute bonne foi qu'ils sont objectifs. Pour le colonisé, l'objectivité est toujours dirigée contre lui. ${ }^{52}$

De la même manière, Rabehl constate que la presse bourgeoise présente les manifestants comme des terroristes, et déploie contre eux tout l'appareillage idéologique réservé aux minorités ${ }^{53}$.

Ce qui est en question ici c'est bien le changement qualitatif du rapport entre politique et morale dans les sociétés occidentales. Si à l'époque de la révolution bourgeoise, chez Kant et Robespierre, la politique contenait un élément inconditionné et une négation catégorique de toute contradiction entre morale et politique, dans les sociétés post-révolutionnaires cette radicalité a été remplacée par le compromis et la transaction. L'intransigeance morale a été progressivement confinée dans la sphère privée, ce qui a entraîné la dépolitisation de l'espace public dont il était question plus haut. Le système de domination avec sa ritualisation de règles et procédures a pour objectif la conservation de sa légitimité et ne produit «qu'une seule forme de sensibilité, notamment celle qui est réceptive aux fonctions de perturbation du fonctionnement de la société, aux manifestations et protestations radicales ${ }^{54}$. Les manifestations qui ne sont pas perçues comme une véritable perturbation sont donc autorisées et permettent de renforcer le système lorsque ce dernier montre sa tolérance démocratique face à ses opposants. En revanche, toute manifestation qui brise et déjoue les règles « démocratiques » et les met en question doit être réprimée sans appel. L'opération de la presse conservatrice face à la mobilisation consiste à taxer les étudiants de « blanquistes », en les présentant ainsi «comme une minorité qui s'auto-isole et qui poursuivrait de façon terroriste ses objectifs élitistes et manipulateurs ${ }^{55}$. Deleuze et Guattari

${ }^{52}$ Ibid., p. 75.

${ }^{53}$ B. RABEHL, « Du mouvement antiautoritaire à l'opposition socialiste », op. cit. p. 341.

${ }^{54}$ O. Negt, Politik als Protest, op. cit. p. 32-33.

${ }^{55}$ R. DutschKe, «Les étudiants antiautoritaires face aux contradictions présentes du capitalisme et face au tiers-monde », op. cit. p. 161. 
suggéreraient que l'État impose sa logique molaire. La finalité est de mobiliser tout le «centre » démocratique, la majorité silencieuse, avec l'appareillage conceptuel qui a marqué tristement l'histoire allemande du XXe siècle : discipline, ordre, paix, espace vital. C'est dans le sillage de ce schéma que la presse Springer caricature le mouvement, la stratégie étant de neutraliser ses meneurs.

Mais à partir de la manifestation contre Tschombé la mobilisation adopte des initiatives qui visent à « déjouer " les règles démocratiques ${ }^{56}$, à contourner les tabous et la logique de capture étatique (interpellation). Afin de « détourner » le contrôle policier et les fictions du droit public, les étudiants découvrent la mobilité et le passage entre d'une part l'espace public, en tant qu'espace autorisé pour une manifestation, à savoir le territoire assujetti au contrôle policier, et de l'autre l'espace privé, externe à la manifestation, où ils redeviennent des citoyens comme les autres. Les étudiants sortent ainsi des manifestations pour se retrouver là où leurs revendications peuvent se faire entendre, c'est-à-dire là où la cible de leur protestation se trouvait, et, bien entendu, où les autorités avaient interdit leur présence. Par ces déplacements ils « déjouent » précisément la séparation fictive entre sphère privée et sphère publique qui est à la base de l'ordre établi. La soi-disant "manifestationpromenade» a en ce sens une valeur emblématique. Il s'agit d'une manifestation non-autorisée (donc techniquement illégale) qui a eu lieu sur la Kurfürstendamm le 17 décembre 1966 lorsque les familles berlinoises sont en train de faire leurs achats de Noël. Les étudiants s'habillent comme de bons citoyens et ils se confondent dans la foule, et, tout d'un coup, ils se regroupent en criant quelques slogans et en distribuant des tracts, pour se disperser de nouveau... Ils « déjouent » ainsi le système d'interpellation policier au point que des 24 arrestations seulement deux concernèrent des étudiants (parmi lesquels Rudi Dutschke, bien connu de la police) $)^{57}$. Par là les étudiants semblent avoir trouvé une originale mise en pratique, ou traduction, des enseignements tactiques de la Guerre de guérilla. Dans cet abécédaire guérillero Ernesto Guevara souligne notamment l'importance de la mobilité et des stratégies pour «détourner» l'ennemi afin de mieux le surprendre. Il explique également que, afin d'assurer sa survie, la bande doit s'adapter et retourner en sa faveur toute circonstance. L'élasticité et l'agilité dans les

${ }^{56}$ Ibid.

${ }^{57}$ Ibid. p. 164. B. RABEHL, « Du mouvement antiautoritaire à l'opposition socialiste », op. cit., p. 349. 
déplacements sont des facteurs cruciaux pour son existence ${ }^{58}$.

C'est sur cette modalité innovante que les étudiants forgent le mot d'ordre de l'«Aufklärung durch Aktion ». Ce slogan détermine la ligne de développement du mouvement, sa ritournelle expansive sur la frontière entre légalité et illégalité. L'action dans la rue a son corrélat dans l'organisation de l'université oppositionnelle avec ses cours auto-gérés et ses contre-cours, qui doivent incarner l'idéal de l'Université critique ${ }^{59}$. Le seul espace public à leur disposition - concrètement : les rues - devient le lieu de dénonciation de la société uni-dimensionnelle et de sur-répression (Marcuse), et l'Université le lieu d'analyse et d'élaboration théorique, de la construction d'une alternative et de l'établissement de stratégies, de tactiques et de techniques de subversion. Les rues et l'Université constituent les deux piliers de l'action du mouvement antiautoritaire. Tous les deux feront l'objet de la répression étatique en 1967 lorsque la police, avec l'appui du recteur, fait irruption dans l'enceinte de l'Université Libre de Berlin et occupe les rues en interdisant toute manifestation.

\section{Le « 2 juin » : le problème de l'organisation et de la violence.}

Après les mobilisations contre la guerre au Vietnam, deux événements en 1967 marquent un transcodage du conflit et un passage qualitatif de l'affrontement. Le premier est « l'attentat au pudding » que les militants de la Kommun 1 préparent contre le vice-président américain Humphrey. Cet épisode témoigne de la tension au sein des forces de l'ordre et des possibles stratégies qu'elles mettent en œuvre pour favoriser la tension sociale et l'escalade de l'affrontement. Dans les pages de Konkret, Meinhof se révolte contre le fait que «ce ne sont ni la terreur ni l'engagement des forces spéciales, qui sont criminalisés, mais les protestations contre ces mesures ». Elle dénonce le point de vue partial et conservateur de la presse.

On peut considérer impoli de frapper un politicien avec du pudding et du fromage blanc ; en revanche il est tout à fait normal d'accueillir des politiciens qui portent la responsabilité de la destruction de villages et des bombardements de la population civile (...). On peut considérer

\footnotetext{
${ }^{58}$ Che Guevara, La guerre de guérilla, Paris, Flammarion, 2010, p. 48-52.

${ }^{59}$ Cf. R. Dutschke, Ecrits Politiques, op. cit., p. 58-60.
} 
impoli de discuter contre l'oppression du peuple vietnamien dans la rue et dans les gares, en revanche on considère tout à fait normal de coloniser un peuple entier sous le couvert de l'anticommunisme. ${ }^{60}$

L'interpellation des communards par les forces de l'ordre se produit de manière grotesque. Ils sont livrés sur la scène médiatique comme des véritables terroristes. La critique de Meinhof ne ménage pas non plus l'attitude des activistes puisque, avec leur comportement auto-référentiel, ils ont perdu une belle occasion de sensibiliser l'opinion publique vis-à-vis des contradictions du système visées par la contestation. Seuls les étudiants se révoltent contre les omissions de ce type d'informations dans la presse et dénoncent la complicité du gouvernement qui a autorisé le survol du territoire par les bombardiers américains en route pour le Vietnam.

L'assassinat de Benno Ohnesorg, le 2 juin, constitue le tournant décisif pour le destin de la mobilisation. Oskar Negt discerne après-coup toute la signification politique de cet événement tragique, en décrivant Benno Ohnesorg comme « la victime d'une action symbolique qui devait prouver la détermination et l'inflexibilité de l'appareil policier ». S'il ne s'agit pas d'un assassinat politique comparable à ceux de Rosa Luxembourg et Karl Liebknecht, meneurs des Spartakistes - puisque Benno Ohnesorg est plutôt «la victime accidentelle d'une action policière planifiée $»^{61}$ - les étudiants perçoivent pourtant cet événement comme la criminalisation définitive de l'opposition et l'indice d'un tournant autoritaire de la société. La lettre ouverte que Ulrike Meinhof adresse à l'épouse du Chah permet de mieux comprendre la «température» politique de ces journées berlinoises. Meinhof accuse Madame Pahlavi d'avoir donné une image fausse de la situation en Iran dans son article paru dans la Neue Revue du 7 et 14 mai et accuse les médias allemands qui cachent l'oppression et la misère de la population persienne sous le régime du Chah de ne pas avoir informé l'opinion public au sujet des financements versés par le gouvernement fédéral pour appuyer le coup d'État contre Mossadegh en $1953^{62}$.

Les étudiants critiquent les autorités publiques parce qu'elles accueillent le Chah sans ressentir l'exigence de prendre leurs distances vis-à-vis de sa

${ }^{60}$ U. MeInHof, Das Wesen des Menschen ist antastbar, op. cit. p. 93.

${ }^{61}$ O. Negt, Politik als Protest, op.cit. p. 25-27.

${ }^{62}$ Certains étudiants distribuent cette lettre comme tract lors de la manifestation (Dokumentation Freie Universität Berlin von 1948-1973, Band V, p. 174). 
politique répressive, en se rendant ainsi complices de ses crimes. Mais paradoxalement, loin de sensibiliser l'opinion publique vis-à-vis du mouvement, la mort d'un jeune étudiant tué par la police le jour de sa première manifestation, renforce l'isolement des étudiants. La réaction des autorités est d'interdire toute manifestation et d'occuper militairement les rues. L'espace médiatique est occupé par les débats sur les lois d'état d'urgence et sur la grande coalition (CDU-SPD). L'accusation de Habermas s'appropriant la thèse de la presse Springer pour taxer les étudiants anti-autoritaires de « fascistes de gauche ${ }^{63}$ illustre bien leur isolement extrême. Selon Oskar Negt, qui interprétait pourtant la déclaration de Habermas comme une simple mise en garde à l'adresse des étudiants qui devaient à tout prix éviter de se couper complètement de la société,

l'accusation de fascisme de gauche est l'expression d'une situation de décomposition de la conscience libérale bourgeoise qui est touchée par la faiblesse des institutions et des règles démocratiques en Allemagne.

Cet état de choses portait à voir « dans l'alternative socialiste seulement la fin de toute liberté $\gg{ }^{64}$.

La mort de Benno Ohnesorg pose la question de la violence et de l'organisation pour faire face à cette montée du degré de l'affrontement. Rudi Dutschke analyse avec lucidité l'atmosphère qui règne au sein de l'Université :

Le 2 juin 1967 est entré dans l'histoire des universités et de la société allemande d'après-guerre, une bonne partie des étudiants s'étaient dressés contre la structure de la base, autoritaire, de la société allemande. Par leurs manifestations, ils l'avaient obligée à montrer son vrai visage, celui de l'autorité irrationnelle. ${ }^{65}$

Le 2 juin fonctionne comme moment de saturation de l'espace d'existence du mouvement. En s'appropriant militairement la rue, la force

${ }^{63}$ Lors d'une rencontre à Hannover le 9 juin 1967, après avoir entendu à la radio l'attaque de Rudi Dutschke contre son « objectivisme », Habermas demanda la parole : «Je suis persuadé qu'il [Dutschke] nous a exposé en l'occurrence une idéologie volontariste qu'on avait taxée en 1848 de socialisme utopique et que dans la conjoncture actuelle - ou du moins je suis persuadé d'avoir des bonnes raisons pour proposer cette terminologie - il faut appeler fascisme de gauche » (J. Miermeister (éd.), Geschichte ist machbar. Texte über das herrschende Falsches und die Radikalität des Friedens, 1980, Berlin, Wagenbach,1991, p. 82).

${ }^{64}$ O. Negt, Politik als Protest, op. cit. p. 85.

${ }^{65}$ R. DutschKe, «Les étudiants antiautoritaires face aux contradictions présentes du capitalisme et face au tiers-monde », op. cit. p. 174. 
publique confine les étudiants dans les enceintes de l'Université. Selon Dutschke « le 2 juin, des troupes entraînées à la guerre civile nous ont réduits au rang d'objets »; ceci ne va pas sans produire une certaine résignation chez les étudiants, au point que la plupart d'entre eux ne croit plus possible de faire de l'Université " une base de combat subversif » dans la société. Mais, ajoute$\mathrm{t}$-il : «Renoncer à l'initiative revient en effet à assumer une attitude passive et résignée, laquelle risque de s'installer pour des semaines et des mois ». Que faire, donc ? L'enseignement de Fanon, de Che Guevara et des guérilleros est qu'il faut maintenir l'initiative, sans pourtant s'engager dans un combat frontal qui serait suicidaire. Parfaitement déployé dans sa version autoritaire, l'Etat impose désormais le niveau d'affrontement maximal : la guerre civile. La police avait exhibé sa puissance lors de l'interpellation des communards qui voulaient simplement lancer comme une nouvelle provocation des « bombes au pudding » sur Humphrey. Selon Dutschke, l'usage de la violence physique produirait un effet contre-révolutionnaire et condamnerait définitivement le mouvement ${ }^{66}$. Mais c'est une fois de plus l'enseignement de Guevara qui guide sa pensée lorsqu'il établit la différence entre sabotage et terrorisme dans la lutte de guérilla. À la différence du sabotage, l'adoption des techniques terroristes peut provoquer un effet contre-révolutionnaire, coupant la guérilla des masses. Dutschke traduit cet enseignement et revendique la lutte antiautoritaire ayant pour fin la conjuration de l'option de la lutte armée :

Il faudrait que, dans le camp anti-autoritaire, les éléments les plus aptes soient chargés de diriger et d'organiser les manifestations, à tous les niveaux. Le soin de diriger les luttes, sous leurs diverses formes, reviendrait donc à des comités d'action, fruits de l'expérience collective et de l'amitié personnelle, dont les membres ne seraient ni des professionnels rémunérés, ni des chefs à qui obéir sans broncher. Ainsi le développement de l'esprit d'initiative et la participation de tous deviendraient-ils à nouveau possibles. Une direction manipulatrice, c'est l'exploitation de manifestants réifiés, la frustration et la résignation, tandis qu'avec une direction émancipatrice l'activité pratique latente, la clarification en profondeur permet à une conscience réelle de s'affirmer. ${ }^{67}$

${ }^{66}$ Une position que Dutschke défend, tant publiquement (cf. R. DutschKe, Ecrits politiques, op. cit., p. 99) que dans ses notes personnelles (cf. R. DuTsCHKE, Jeder hat sein Leben ganz zu leben, op. cit. p. 44).

${ }^{67}$ R. DutschKe, «Les étudiants antiautoritaires face aux contradictions présentes du capitalisme et face au tiers-monde » op. cit. p. 176-177. 
L'engagement doit être total. Le révolutionnaire métropolitain doit se consacrer lui aussi complètement à la lutte.

Que «le temps du jeu provocateur est fini », veut dire pour Dutschke qu'a été « atteint le point où les œufs pourris ne suffisent plus ». Le mouvement doit redéfinir sa stratégie face au transcodage imposé par les forces de l'ordre. Si œufs et tomates avaient été nécessaires pour attirer l'attention de l'opinion publique, « la politisation avait [désormais] atteint une phase où il serait stupide de recourir à de telles méthodes, qui d'ailleurs nous feraient perdre le terrain que nous avons gagné ». Les possibilités de développement du mouvement anti-autoritaire reposent alors sur sa capacité à se transformer en machine de subversion s'agençant avec d'autres milieux. Cela implique la formulation de nouvelles stratégies d'action que Dutschke et Krahl élaborent en traduisant le modèle alternatif de la guérilla à la base de la révolution cubaine et d'une possible révolution en Amérique Latine comme «agitation permanente » dans les métropoles et dans les institutions des démocraties occidentales. Il faut faire face à l'absence d'un sujet révolutionnaire classique - au fur et à mesure que la majorité de la classe ouvrière s'intègre à l'appareil d'Etat - par des nouvelles pratiques de subjectivation politique, c'est-à-dire qu'il faut redéfinir l'horizon pour développer des subjectivités subversives.

Rudi Dutschke et Hans-Jürgen Krahl intitulent leur intervention au 22ème congrès du SDS (septembre 1967) : "Pratiquer le refus implique une mentalité-guérilla ». Lors de ce congrès le SDS se trouve obligé pour la première fois après sa sortie du SPD de redéfinir sa ligne politique. La scène politique est marquée par deux événements majeurs : la Grande Coalition (réunissant les deux partis populaires: CDU et SPD) et les effets de l'assassinat de Benno Ohnesorg qui réduisent au minimum les possibilités d'action des militants. Par leur intervention, les deux meneurs du mouvement essaient de concilier deux positions : celle du SDS berlinois qui après l'assassinat de Benno Ohnesorg propose la formation de comités d'action dans les quartiers pour passer à une politisation de la ville, et celle d'une grande partie du SDS, qui propose une alliance extra-parlementaire, une sorte de front avec les autres groupes de la gauche radicale pour organiser l'opposition à la grande coalition CDU-SPD.

Dutschke et Krahl proposent que :

Les groupes de conscience révolutionnaires (revolutionäre 
Bewußtseinsgruppen) qui en raison de leur position spécifique dans les institutions peuvent donner concrètement des signaux oppositionnels capables d'éclairer la situation, doivent utiliser une méthode de lutte politique qui se distinguerait en principe des formes traditionnelles de la confrontation politique. ${ }^{68}$

Ils sont bien conscients de l'enjeu théorique de leur discours. Et ils poursuivent :

L'agitation dans l'action et l'expérience des combattants solitaires dans la confrontation avec le pouvoir exécutif étatique forment des facteurs de mobilisation pour l'extension de l'opposition radicale ; et rendent tendanciellement possible un processus de prise de conscience pour des minorités actives au sein des masses passives. Celles-ci ne peuvent s'apercevoir de la violence abstraite qu'à travers l'action irrégulière [des minorités]. Il faut que la «propagande des coups » dans le tiers-monde se complète dans les métropoles par la «propagande de l'acte » qui rend possible une urbanisation de l'activité de la guérilla rurale. Le guérillero urbain est l'organisateur d'irrégularités comme destruction [immanente] du système des institutions répressives. ${ }^{69}$

En ce qui concerne la base-arrière - la zone de sécurité nécessaire au développement de la guérilla, notamment la campagne - les deux théoriciens indiquent qu'elle doit être l'Université en tant que « base sociale, dans laquelle et à partir de laquelle le guérillero doit organiser sa bataille pour le pouvoir dans l'Etat». Et à la question : « Pourquoi proposer cela au SDS ? » ils répondent qu'ils sont bien conscients que «plusieurs camarades ne sont plus disposés à accepter un socialisme abstrait qui n'a rien à voir avec leur propre activité vitale en tant qu'attitude politique ». En somme, dans le SDS il existe des présupposés personnels pour une nouvelle forme de coopération dans les groupes. «Le refus dans les milieux des institutions nécessite une mentalitéguérilla, si on ne veut pas tomber dans l'intégration ou dans le cynisme $\gg^{70}$. Bref : la seule issue possible face à l'alternative imposée par l'Etat après le 2 juin - intégration ou résignation - est un refus organisé selon la modalité de la guérilla comme agitation. Ainsi s'ouvre « la longue marche dans les institutions » qui doit se faire par un patient travail de mobilisation de base à

${ }^{68}$ H-J. KRAHL/ R. DuTSChKe, Das Sich Verweigern erfordet Guerilla-Mentalität in J. Miermeister (éd.), Geschichte ist machbar, op. cit. p. 94.

${ }^{69}$ Ibid.

${ }^{70}$ Ibid. p. 95. 
l'intérieur des syndicats, des partis et de toute institution. Il faut devenir agitateurs politiques dans chaque discipline et dans chaque domaine de la vie sociale.

Face au déclin économique et politique de Berlin-Ouest, Dutschke propose dans une autre intervention des actions analogues aux «bases rouges » que « Potere Operaio » réalisera en Italie quelques années plus tard. Il imagine la constitution dans les quartiers de comités pour des luttes concrètes, par exemple contre l'augmentation des prix des loyers, contre le Groupe Springer, etc. Refuser cette société signifie pour lui en organiser autrement les intérêts, les désirs et les souffrances; les réorganiser par des pratiques immanentes capables de désarticuler de l'intérieur les rapports de verticalité et de soumission jusqu'à rendre superflu tout appareil politique. Il faut concrètement occuper la ville par-delà les manifestations, et mobiliser d'autres couches de la population par des batailles concrètes dans les quartiers. Cette mobilisation devrait faire de Berlin-Ouest une société libre, une nouvelle Commune au cœur de l'Europe divisée. À ce processus aurait dû, en même temps, s'articuler une dissolution des comités d'action qui avaient soutenu et rendu possible la mobilisation étudiante afin d'éviter qu'ils se transforment en une nouvelle élite suivant le modèle léniniste d'un parti éducateur du peuple et des masses. Leurs membres devaient s'intégrer au travail des AG. C'est par le biais des comités de surveillance des prix et des loyers, des comités d'orientation qui dispensaient des renseignements juridiques, économiques et pratiques pour aider et politiser d'autres milieux, que Dutschke voulait réaliser un «Berlin soutenu par le bas », c'est-à-dire par une démocratie des soviets directs où les chefs en rotation perpétuelle seraient élus par des individus libres, et choisis dans les entreprises, les écoles et universités, l'administration etc. ${ }^{71}$. Nous avons déjà remarqué que le rêve de Dutschke était de faire de Berlin la patrie du véritable socialisme et la base pour la réunification de l'Allemagne et de l'Europe.

Avant de liquider trop rapidement ce discours comme utopique, il nous faut prendre en compte l'effet de la révolution cubaine et la force de son mythe. À savoir l'idée d'une petite cellule qui petit à petit grandit et forme une armée révolutionnaire forçant une dictature à la déroute. Ce mythe joue un rôle important dans ce type de discours et dans la théorisation d'une phaseguérilla pré-révolutionnaire en Europe Occidentale. En outre, la base affective

\footnotetext{
${ }^{71}$ Cf. R. DutschKe, Ecrits politiques, op. cit., p. 146-154.
} 
de ce discours est la conviction que la révolution est un choix qui implique un dévouement total, qu'elle doit être une révolution culturelle ou, selon les paroles de R. Debray, une « révolution dans la révolution ». Selon Negt « la résistance permanente contre la fonctionnalisation du savoir au service des intérêts dominants constitue [l'essence de l'expérience de l'] université [critique]». Elle est soutenue par «l'exigence d'articuler les intérêts émancipateurs des êtres humains dans un quatrième pouvoir autonome et de s'attaquer activement au processus de formation de la volonté de la société $\rangle^{72}$. Autrement dit, la lutte doit s'attaquer à chaque domaine de la vie, il n'existe plus de séparation fictive entre privé et public, de division artificielle du savoir dans des disciplines. Pour mettre fin à tout cela, la politique doit renouer avec la vérité et la justice. Dutschke avait d'ailleurs explicité à maintes reprises sa position personnelle par rapport à une forme quelconque de résistance armée. Il considérait celle-ci comme étant contre-révolutionnaire au sein des métropoles occidentales, où on devait plutôt conduire une bataille pour l'Aufklärung de la société, et viser le lien entre la paix interne dont jouissent les démocraties occidentales et les guerres dans les pays du tiers-monde. Il insistait sur le fait qu'il s'agissait moins d'une attitude morale que d'une position purement tactique. Il avait affirmé sans équivoque qu'il aurait bien pris les armes s'il s'était trouvé dans les pays du tiers-monde. Il voyait encore de l'espace politique pour une action qui ne s'attaque pas à la vie et au corps de l'adversaire - une valeur encore sacro-sainte dans les démocraties occidentales.

\section{L'attentat contre Dutschke : « Exproprier Springer !».}

Cinq mois après avoir ainsi exposé ses ambitions révolutionnaires, le jeudi de Pâques 1968, Rudi Dutschke est victime d'un attentat qui l'éloignera de la scène politique allemande pendant des années. La réaction des étudiants est violente et vise immédiatement Springer, en donnant l'assaut à des fourgonnettes chargées de distribuer le Bild-Zeitung à Berlin et en mettant le feu aux journaux. Leur réaction est lucide et s'attaque de façon cohérente à ceux qu'ils considèrent comme les véritables responsables de l'attentat. Dans son article « Rechtsordnung, Öffentlichkeit und Gewaltanwendung », Oskar

${ }^{72}$ O. NeGt, Politik als Protest op. cit., p. 49. 
Negt souligne que cette action n'est pas symbolique mais bien plutôt «l'expression spontanée et manifeste de la résistance pratique contre une entreprise éditoriale avec laquelle aucun mouvement véritablement démocratique ne peut coexister longtemps ». La réaction face à l'attentat ne vise pas la police de façon " hystérique », mais agit " consciemment contre une entreprise qui est perçue au moment de l'attentat comme un outil de la violence de l'ordre établi $\gg^{73}$. À la différence de l'affaire Ohnesorg, lors de laquelle avaient été prises en compte les circonstances atténuantes « objectives » afin de pouvoir acquitter l'agent Kurras, les autorités rejettent toutes les responsabilités sur Bachmann, le militant d'extrême droite auteur de l'attentat, en ignorant délibérément tout effet objectif des circonstances, notamment la campagne de presse féroce et diffamatoire engagée par Springer contre Dutschke, qui renvoyait systématiquement à toutes les figures perçues dans l'imaginaire populaire comme dangereuses pour la démocratie (Hitler, Staline etc.). Selon Negt, les étudiants avaient fait preuve de lucidité, car les véritables responsables sont bien les journalistes de Springer et leurs méthodes que Günther Grass avait taxées de fascistes. C'est à partir de là que se poursuit la campagne pour l'expropriation de Springer. À la question «Pourquoi exproprier Springer?» Ulrike Meinhof répond ainsi sur les colonnes de Konkret :

Parce que toute tentative de re-démocratisation de ce pays, de reconstitution d'une volonté populaire et de formation de citoyens capables de juger la réalité échoue et ne pourra qu'échouer si Springer conserve la force qu'il a maintenant. Non pas parce qu'il abuse de cette force, mais simplement parce qu'il en dispose. ${ }^{74}$

Après l'attentat contre Dutschke, la question de l'organisation de la contre-violence et de la résistance armée se pose alors d'une façon encore plus radicale. Dans ses articles pour Konkret, Ulrike Meinhof y voit un changement, le franchissement d'un seuil, dans le combat, voire le basculement définitif de la protestation dans la résistance :

C'est fini le divertissement. Protestation, c'est lorsqu'on dit que ceci et cela ne nous convient pas. Résistance, c'est quand je fais tout le possible afin que ce qui ne me convient pas, ne dure pas plus longtemps. ${ }^{75}$

\footnotetext{
${ }^{73}$ Ibid. p. 27.

${ }^{74}$ U. MEInHof, Die Würde des Menschen ist antastbar, op. cit., p. 105.

${ }^{75}$ Ibid., p. 140.
} 
La question du droit de résistance relancée par Marcuse dans son essai sur la Tolérance répressive, subit alors un transcodage par les événements. Certains considèrent que le jour est désormais venu de déclencher la guérilla armée dans les métropoles. À vrai dire, quelques jours avant l'attentat contre Dutschke, quatre militants berlinois (dont Andreas Baader et Gudrun Ensslin), étaient déjà passés à l'acte en provoquant un incendie sans victimes dans un grand magasin de la chaîne Kauf-Hof à Francfort. Ils répondaient ainsi à l'appel de la Kommun 1 qui, après un attentat incendiaire à Bruxelles, avait demandé de façon provocatrice : «Quand brûleront finalement les grandsmagasins à Berlin-Ouest ? ». La police les interpelle quelques jours plus tard, et leur procès devient un véritable happening politique. Les juges accordent aux inculpés que leur action avait des raisons politiques et ne les condamnent qu'à quelques mois de prison. Si ce n'est pas cet épisode l'acte fondateur de la RAF, c'est pourtant bien dans les chroniques de ce procès que les noms d'Andreas Baader et Gudrun Ensslin vont faire la une des journaux pour la première fois, en se faisant connaître comme activistes radicaux.

C'est à partir de l'échec du mouvement allemand qui s'ouvre l'option suicidaire de la lutte armée. Dans le texte « Concept de guérilla urbaine » de la RAF, Meinhof le reconnaîtra explicitement :

Si le mouvement étudiant pouvait nommer les buts et contenus de la lutte anti-impérialiste (...) [il] n'était pas lui-même le sujet révolutionnaire, ne pouvant pas se permettre la médiation organisationnelle. À la différence des « organisations prolétaires » de la nouvelle gauche, la Fraction Armée Rouge ne nie pas sa préhistoire comme histoire du mouvement étudiant, qui a reconstruit le marxismeléninisme comme arme dans la lutte de classe et a posé le contexte international pour le combat révolutionnaire dans les métropoles. ${ }^{76}$

Tout en soulignant la « césure désastreuse » et sclérosante que la lutte armée a provoqué dans ses formes terroristes tant étatique que groupusculaire, Félix Guattari et Toni Negri remarquent que les États ont imposé aux mouvements de contestation des années 1970 un affrontement molaire qui a sapé toute subjectivation dissidente et existentielle. De son côté, le terrorisme rouge européen a relancé des conceptions et des idéologies de l'organisation

${ }^{76}$ Rote Armee Fraktion, Le concept de Guérilla urbaine (1971) in M. Hoffmann (éd.) Rote Armee Fraktion. Texte und Materialien zur Geschichte der RAF, Berlin, ID Verlag, 1997, p. 2748 (tr. fr. in whL : http://labourhistory.net/raf/documents/fr/0019710501\%20FR.pdf ). 
centralisée qui étaient sans aucun doute myopes non moins qu'obsolètes.

Sa folle recherche de points centraux d'affrontement est entrée en redondance avec un léninisme ossifié, déconnecté de tout Phylum historique, entièrement réduit à une interprétation étatique, sorte de référence paranoïaque qu'il prétendait imposer à la recomposition de la subjectivité prolétarienne. ${ }^{77}$

Ce terrorisme n'a « qu'un destin, celui de l'échec et du désespoir », et il constitue une véritable ligne de mort pour toute dynamique d'émancipation. Cependant Guattari et Negri affirment que :

il faut reconnaître que cette vague terroriste a posé un problème vrai à travers des prémisses et des réponses fausses: comment lier la résistance contre la réaction à la mise en place d'un nouveau type d'organisation ? ${ }^{78}$

${ }^{77}$ F. Guattari et A. Negri, Les nouveaux espaces de liberté, Paris, Lignes, 2010, p. 90-91.

${ }^{78}$ Ibid. 